\title{
Neue Institute und umweltrelevante Studiengänge
}

\section{Umweltrelevante Studiengänge \\ - Übersicht der Gesamtsituation im deutschsprachigen Bereich}

Umweltwissenschaftliche Studiengänge erfreuen sich großen $\mathrm{Zu}$ laufs, und ständig erweitern die Hochschulen ihr Angebor auf diesem Gebiet. Es gibt wohl keinen Studiengang mehr, der von dieser Entwicklung unberührt geblieben ist. Naturwissenschaftliche und technische Fächer machten den Anfang, in den letzten Jahren zogen vermehrt gesellschaftswissenschaftliche Fächer nach - leider meist immer noch auf getrennten Wegen.

Die in dieser Ausgabe der Zeitschrift beginnende Artikelreihe gibt einen Überblick übcr umweltbezogene Studienangebote im deutschsprachigen Raum. Der erste Teil stellt die Gesamtsituation dar, es folgen Beschreibungen der einzelnen Studiengänge $(\rightarrow$ Tabelle). In Ausgabe 1/91 (Januarheft) wird das "Weiterbildende Studium Umweltberatung" der Universität Bielefeld vorgestellt (s.a. $\rightarrow$ S. 179 , Ausgabe 3/90 "Dritte Bielefelder Aktuelle Woche zur Umweltberatung").

\section{Bundesrepublik Deutschland (ehemalig)}

Der vom Umweltbundesamt herausgegebene „Studienführer Umweltschutz" verzeichnet 97 Voll- und 18 Aufbaustudiengänge an 46 Universitiaten sowie 73 Voll- und drei Aufbaustudiengänge an 51 Fachhochschulen.

Größtenteils sind dies allerdings mehr oder weniger „klassische“ Studiengänge mit umweltspezifischen Fachrichtungen im Hauptstudium, beispielsweise Biologie, Verfahrenstechnik, Architektur oder Volkswirtschaftslehre. Solche Studienangebote sollen nicht Thema der Artikelserie sein und werden deshalb auch in der Übersichtstabelle nicht aufgeführt (nähere Angaben über diese Studiengänge siebe $\rightarrow$ Studienführer Umweltschutz).

Vielmehr sollen hier die seit Anfang der siebziger Jahre entstandenen eigenständigen Umwelt-Studiengänge vorgestellt werden, wenn auch die Abyrenzung in dem einen oder anderen Fall willkürlich erscheinen mag. Hier wird das Angebor Jahr für Jahr vielfältiger. Im Gegensatz zu den klassischen sind die neuen Studiengänge oft interdisziplinär angelegt, im Überschneidungsbereich Naturwissenschaften-Technik, Naturwissenschaften-Planung oder Gesellschaftswissenschaften-Planung. Es gibt aber - abgesehen von dem Aufbaustudiengang Europäisches Diplom in Umweltwissenschaften - leider immer noch kein interdisziplinäres umweltbezogenes Studienangebot, das gesellschaftswissenschaftliche mit technischen oder naturwissenschaftlichen Fächern verbindet.

\section{Deutsche Demokratische Republik (ehemalig)}

Die Situation in der ehemaligen DDR ist gegenwärtig noch etwas unübersichtlich und wird zu einem späteren Zeitpunkt ausführlich dargestellt:
1. An der Universität Leipzig wurde auf Betreiben des Fachverbandes "Chemische Toxikologie" der Chemischen Gesellschaft ein dreijähriges Postgradualstudium „Toxikologie" für Hochschulabsolventen außerhalb der Medizin eingerichtet, in dessen Ausbildungskonzept die Umwelttoxikologie integriert ist.

2. Die Universität Leipzig bietet weiterhin interdisziplinäre Seminare für den wissenschaftlichen Nachwuchs an, z.B. den Kurs „Ökologische Chemie“ am 8. -12. Oktober 1990.

\section{Österreich}

In Österreich gibt es keine umweltbezogenen Vollstudiengänge, wenn auch einige Hochschulen Kurse und Studienschwerpunkte zu naturwissenschaftlichen, planerischen oder gesellschaftswissenschaftlichen Umweltthemen anbieten.

Die Technische Universität Graz und die Technische Universität Wien gemeinsam mit der Universität für Bodenkultur Wien bieten einen Aufbaustudiengang Technischer Umweltschutz an.

\section{Schweiz}

1. Als einzige deutschsprachige Universität bietet derzeit die ETH Zürich einen umweltbezogenen Vollstudiengang an: Umweltnaturwissenschaften. Dies ist im gesamten deutschsprachigen Raum der ,interdisziplinärste“ Studiengang: 69 Prozent Naturwissenschaften und Mathematik, je 17 Prozent Sozialwissenschaften und Technik sowie 14 Prozent multidisziplinäre Veranstaltungen und Fallstudien.

Die ETH Zürich plant darüber hinaus einen Aufbaustudiengang Umweltwissenschaften und Umwelttechnik.

2. Die Universität Zürich bietet ein einjähriges Nachdiplomstudium Umweltlehre an und plant die Einführung eines weiteren postgradualen Umweltstudienganges, der möglicherweise auch berufstätigen Fachleuten offenstehen wird.

3. Die Universität Bern plant einen problem- (nicht: disziplin-) orientierten Aufbaustudiengang Allgemeine Ökologie.

Daneben besteht ein breitgefächertes Angebot an umweltbezogenen Wahlschwerpunkten oder Teilcurricula für alle Fächer.

\section{Literatur}

Bundesrepublik Deutschland: Umweltbundesamt (Hrsg.): Studienführer Umweltschutz, 4. Auflage, Berlin 1988

Österreich, Schweiz: Institut für Europäische Umweltpolitik: Umweltbezogene Bildungsangebote an Hochschulen ausgewählter Länder, Band 1 (Zusammenfassender Bericht), S. 26-33; Bonn, Dezember 1988 
Übersichtstabelle: Umweltbezogene Studiengänge in der ehemaligen Bundesrepublik Deutschland (Quelle: Studienführer Umweitschutz)

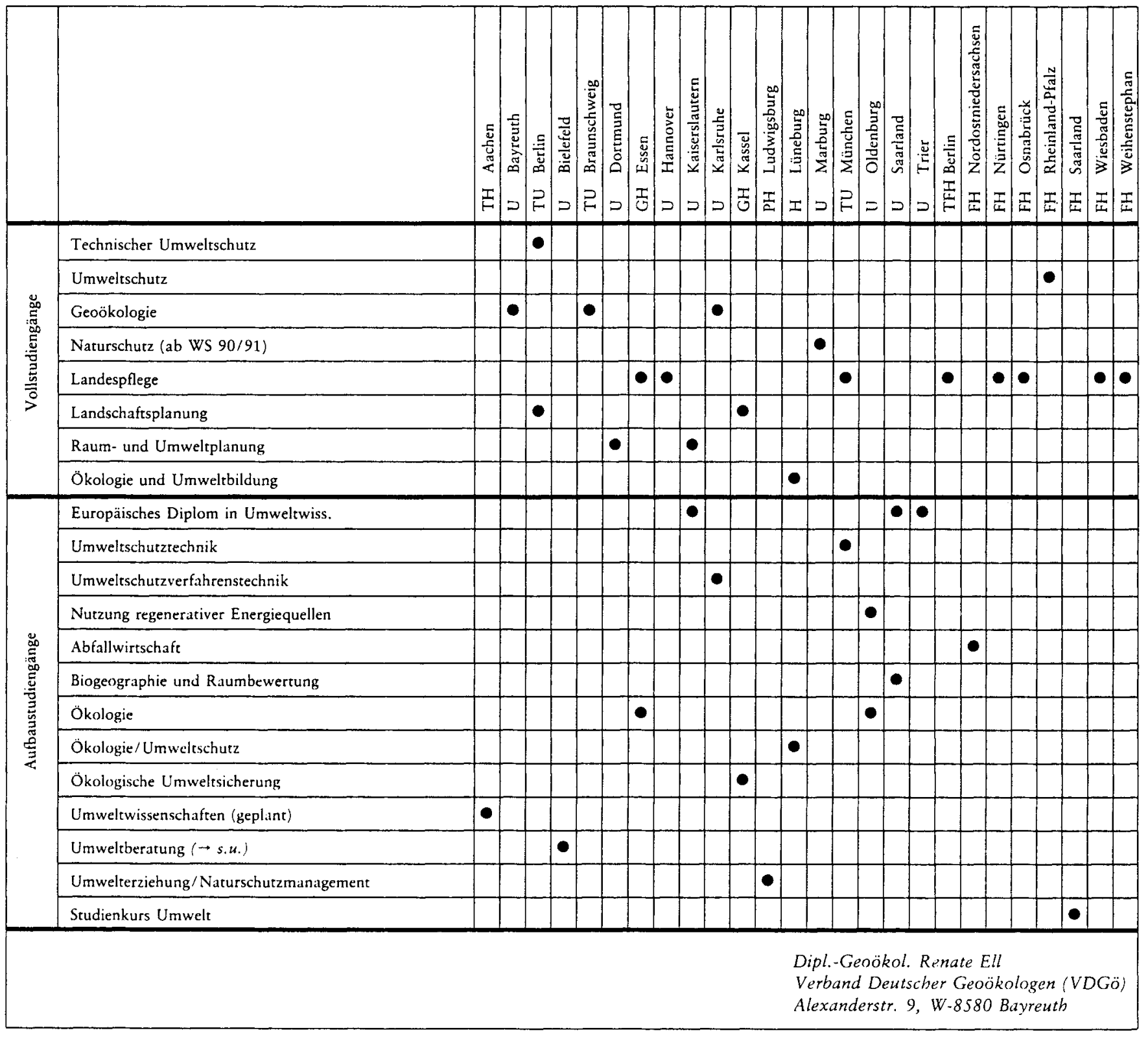

Weiterbildendes Studium Umweltberatung Bielefeld

- Zusätzliche Kurse im WS 1990/91

\section{Titel/Veranstalter}

Organisation des betrieblichen Umweltschutzes

Sicherheitsmanagement und Umwelthaftung

Finanzierung von Umweltinvestitionen und öffentliche Förderung

Ökobilanz und Stoffbilanz

Toxikologie der Innenräume

Umweltschutz im Großhaushalt

EDV im Umweltschutz - Datenbanken und Anwendersoftware

\section{Anmeldungen und Rückfragen:}

Hans-Peter Obladen, Universität Bielefeld

Kontaktstelle Wissenschaftliche Weiterbildung

Weiterbildendes Studium Umweltberatung

\section{Termine/Uhrzeiten}

02. 11.1990

09. 11.1990

16. 11.1990

23. 11.1990

29. 11. $(13.00-17.00 \mathrm{Uhr})$ und

30. 11. 1990 ( 9.00 bis 18.00$)$

11. 01.1991 (11.00-18.00 Uhr)

18. $01.1990(11.00-18.00 \mathrm{Uhr})$

\section{Postfach 8640}

4800 Bielefeld 1

Tel. $0521 / 106-4473$

Fax: 106-5844
Gebühren/Entgelte

$150,00 \mathrm{DM}$

$150,00 \mathrm{DM}$

$150,00 \mathrm{DM}$

$150,00 \mathrm{DM}$

$150,00 \mathrm{DM}$

150,00 DM

$150,00 \mathrm{DM}$ 